\title{
Article
}

\section{Systematic Review and Meta-Analysis of the Effectiveness of Mental Practice for the Upper Limb After Stroke: Imagined or Real Benefit?}

Stockley, Rachel, Jarvis, Kathryn, Boland, Paul and Clegg, Andrew

Available at http://clok.uclan.ac.uk/35993/

Stockley, Rachel ORCID: 0000-0003-4441-6860, Jarvis, Kathryn ORCID: 00000001-5963-7346, Boland, Paul ORCID: 0000-0003-2267-4295 and Clegg, Andrew ORCID: 0000-0001-8938-7819 (2020) Systematic Review and MetaAnalysis of the Effectiveness of Mental Practice for the Upper Limb After Stroke: Imagined or Real Benefit? Archives of Physical Medicine and Rehabilitation . ISSN 0003-9993

It is advisable to refer to the publisher's version if you intend to cite from the work. http://dx.doi.org/10.1016/j.apmr.2020.09.391

For more information about UCLan's research in this area go to http://www.uclan.ac.uk/researchgroups/ and search for <name of research Group>.

For information about Research generally at UCLan please go to http://www.uclan.ac.uk/research/

All outputs in CLoK are protected by Intellectual Property Rights law, including Copyright law. Copyright, IPR and Moral Rights for the works on this site are retained by the individual authors and/or other copyright owners. Terms and conditions for use of this material are defined in the policies page. 
1 Title: Running Head: Mental practice for the upper-limb

2 Title: A systematic review and meta-analysis of the effectiveness of mental practice

3 for the upper-limb after stroke: Imagined or real benefit?

4 Authors:

5 Rachel C Stockley, $\mathrm{PhD}^{1}$

6 Kathryn Jarvis, $\mathrm{PhD}^{1}$

7 Paul Boland, $\mathrm{MSc}^{1}$

8 Andrew J Clegg, $\mathrm{PhD}^{1}$

9 Faculty of Health and Wellbeing, University of Central Lancashire, Preston, UK. PR1

$10 \quad 2 \mathrm{HE}$

11 Corresponding author: Dr R Stockley, Faculty of Health and Wellbeing, University of

12 Central Lancashire, Preston, UK. PR12HE email rstockley1@uclan.ac.uk; Tel 0044

$13 \quad(0) 1772894998$

14 Part of this material was presented at the UK Stroke Forum 2019, Telford, UK.

15 Funding: This work was unfunded; AC is part-funded by the National Institute for Health Research Applied Research Collaboration North West Coast (NIHR ARC

17 NWC). The views expressed are those of the author(s) and not necessarily those of

18 the NIHR or the Department of Health and Social Care.

19 The authors have no conflict of interest.

Acknowledgements:

21 The help of J. Reed and C. Harris, information specialists, is acknowledged for 22 undertaking database searches. 
24 This review was registered with Prospero database of systematic reviews (Prospero 25 reference number CRD42019126044).

26

27

28 Running Head: Mental practice for the upper-limb

29 Title: A systematic review and meta-analysis of the effectiveness of mental practice 30 for the upper-limb after stroke: Imagined or real benefit?

31 The authors have no conflict of interest.

32 This review was registered with Prospero database of systematic reviews (Prospero 33 reference number CRD42019126044).

34 Word count: 4769 
A systematic review and meta-analysis of the effectiveness of mental practice for the upper-limb after stroke: Imagined or real benefit?

\section{Abstract}

Objective: This systematic review sought to determine the effectiveness of mental practice (MP) upon the activity limitations of the upper-limb in people after stroke, and when, in whom and how it should be delivered.

Data sources: Ten electronic databases were searched from November 2009 to May 2020. Search terms included: Arm; Practice; Stroke Rehabilitation; Imagination; Paresis; Recovery of Function; Stroke

Studies from a Cochrane review of MP (up to November 2009) were automatically included. The review was registered with Prospero database of systematic reviews (Reference number: CRD42019126044).

Study selection: Randomised controlled trials of adults after stroke using MP for the upper-limb were included if they compared to usual care, conventional therapy or no treatment and reported activity limitations of the upper-limb as outcomes. Independent screening was carried out by two reviewers.

Data extraction: One reviewer extracted data using a tool based upon the Template for Intervention Description and Replication. Data extraction was independently verified by a second reviewer. Quality was assessed using the PEDro tool.

Data Synthesis: Fifteen studies $(n=486)$ were included and $12(n=328)$ underwent meta-analysis. MP demonstrated significant benefit upon upper-limb activities compared to usual treatment (standardised mean difference, SMD: $0.6,95 \%$ 
59 confidence intervals, $\mathrm{Cl}: 0.32$ to 0.88$)$. Sub-group analyses demonstrated that MP

60 appeared most effective in the first 3 months after stroke (SMD: $1.01,95 \% \mathrm{Cl}: 0.53$

61 to1.50) and in people with the most severe upper-limb deficits (weighted mean

62 difference, WMD: $7.33 ; 95 \% \mathrm{Cl}: 0.94$ to 13.72$)$.

63 Conclusions: This review demonstrates that MP appears effective in reducing

64 activity limitations of the upper-limb after stroke particularly in people in the first three

65 months after stroke and in those with the most severe upper-limb dysfunction. There

66 was no clear pattern of the ideal dosage of MP.

67 Word count: Abstract: 286; Manuscript: 4769

68 Keywords: mental practice; imagery; stroke; systematic review; upper limb

69 List of abbreviations:

70 Confidence interval, $\mathrm{Cl}$

71 Mental Practice, MP

72 Standardised mean difference, SMD

73 Weighted mean difference, WMD 


\section{A systematic review and meta-analysis of the effectiveness of mental practice}

\section{for the upper-limb after stroke: Imagined or real benefit?}

Stroke is the single main cause of acquired disability in high income countries. ${ }^{1}$

Difficulties in using the arm, wrist and hand (the upper-limb) is the most common deficit after stroke, being reported by at least $70 \%$ of stroke survivors. ${ }^{2}$ This has a significant impact on daily activities, and has been shown to reduce independence, the likelihood of returning to employment and hobbies, and poorer mental health and quality of life..$^{3,4}$

Mental practice (MP) is one of only a handful of interventions included in evidence based guidelines for the rehabilitation of the upper-limb after stroke. ${ }^{5,6}$ It comprises the repeated practice of motor (or kinaesthetic) imagery. ${ }^{7}$ During MP participants are typically guided to cognitively rehearse, but not physically perform, movements of the upper-limb often to complete a functional task ${ }^{8,9}$ or to consider how one might perform a task (e.g. grip a cup). ${ }^{10}$ This can be from a first person perspective (egocentric, through one's own eyes) or a third person perspective (as an observer watching from a distance). ${ }^{11}$ Mental practice was initially developed in sports psychology to improve performance, and has been used in both cognitive and physical therapies. ${ }^{12,13}$ Whilst the precise mechanisms by which MP may work have not been fully elucidated, it is agreed that mental imagery utilises stored multimodal (motor and sensory) representational formats and/or previous experiences of movements. ${ }^{14}$ It has been shown that MP activates many of the same areas of the brain that are stimulated when physically executing a movement. ${ }^{15,16}$ These include the premotor cortex, basal ganglia and cerebellum and associative parietal cortex. 
7,15 Consequently MP may provide a 'back door' to the motor cortex as it facilitates motor cortex activity and neuroplasticity without physical movement. ${ }^{15,17-19}$

Studies indicate that the majority of people can undertake MP within the first six weeks after stroke. This does vary depending upon the test used to assess MP ability, ${ }^{16,20,21}$ but the ability to undertake MP after stroke does not appear to be significantly influenced by age. ${ }^{22}$ It is also one of very few treatments that can be used by those who have no voluntary movement of their upper-limb. This enables participation in an upper-limb rehabilitative intervention for those who could not undertake exercise based interventions for the upper-limb, such as constraint induced movement therapy or repetitive task practice. ${ }^{23,24}$ Conversely, its use in high performance sport indicates that it is suitable to be used by people with good upperlimb function to refine high-level skills after stroke. ${ }^{7}$ Crucially, as MP does not require any actual physical movement, it is safe for people after stroke to undertake with only minimal or no supervision. This means that if MP can be shown to be effective, it could provide multiple practice opportunities and be a useful method to supplement the amount of therapist-provided rehabilitation for the upper-limb after stroke and improve outcomes.

Several trials of mental practice for the upper-limb after stroke have shown it to be as effective as some forms of physical practice upon impairments, and it appears particularly efficacious if used alongside physical therapy. ${ }^{8,9,25,26}$ However, others have reported no differences in activity limitations when compared to usual care interventions, ${ }^{27}$ suggesting that its effectiveness cannot be assumed. The most recent Cochrane review of MP for the upper-limb after stroke was published in $2020^{8}$ and found that MP had a significant benefit upon upper-limb motor recovery and activities. Further reviews largely support this finding, ${ }^{28,29}$ although the magnitude of 
the effect appears to differ between studies. Even with the increase in available trials, there is still uncertainty as to whether the changes elicited by MP could specifically reduce activity limitations of the upper-limb, a recognised and shared priority for people after stroke and clinicians ${ }^{30}$. Furthermore, the optimal parameters of use of MP for the upper-limb remain unclear. Specifically, the time period after stroke during which MP might be most effective (when) and the effect of dose upon activity limitations (how much) ${ }^{31}$ have not been identified.

Therefore, this review seeks to address this shortcoming by:

(i) examining the effectiveness of MP upon outcomes that specifically measure activities and activity limitations,

(ii) describing when and in whom after stroke MP might have most benefit to upper-limb activity outcomes

(iii) investigating if and how the dose affects the effectiveness of MP

The lack of clear guidance regarding how and in whom MP should be used clinically may, in part, explain why despite its inclusion in stroke guidelines, MP is reported to be rarely used in practice. ${ }^{32}$ The information generated by this review will provide greater clarity for clinicians regarding how they might choose to use MP in practice and identify clear indications of priorities for future research.

\section{Methods}

This review follows the Cochrane Reviews of mental practice and utilised the same search criteria. ${ }^{8}$ It was registered with Prospero database of systematic reviews (Prospero reference number: CRD42019126044) and followed published checklist and guidance on systematic reviews (PRISMA and Cochrane). ${ }^{33,34}$ 
147 Electronic searches of the following databases were completed: Cochrane Central

148 Register of Controlled Trials (CENTRAL), MEDLINE, EMBASE, CINAHL,

PsycINFO, Scopus, Web of Science, the Physiotherapy Evidence Database (PEDro)

150 (http://www.pedro.org.au/), the specialist rehabilitation research databases CIRRIE 151 (http://cirrie.buffalo.edu) and REHABDATA (www.naric.com). The databases were searched from the point of the last Cochrane review (November 2009) until $4^{\text {th }}$ May 2020.

154 Search terms included: Arm; Practice; Stroke Rehabilitation; Imagination; Paresis;

155 Recovery of Function; Stroke

The search strategy is documented in appendix 1.

\section{Screening and Selection}

158 Titles and abstracts were independently screened by two reviewers (RS and KJ).

Studies were included if: they were a parallel group randomised controlled trial; participants were over16 years of age with a confirmed diagnosis of stroke (clinical criteria and/or scanning) and had a sensorimotor upper-limb involvement as a result of their stroke; compared a MP intervention, defined as cognitive rehearsal of a movement or task for the upper-limb, ${ }^{9}$ to conventional therapy, usual care, a defined placebo intervention or no therapy; and the effects of MP could be delineated from other interventions. Only studies whose full text was available in English and that used outcomes that measured upper-limb activities before and after the intervention were included. Upper-limb activities were defined according to the $\mathrm{WHO}$ criteria ${ }^{35}$ and included lifting/carrying and putting down (d4300,4301, 4302, 4305) fine hand use (d440) and hand and arm use (d445). 
After title and abstract screening, the full text of selected studies were retrieved, independently read and assessed for inclusion. Any papers where suitability was unclear were reviewed by two reviewers (RS and $\mathrm{KJ}$ ) and a decision made through discussion.

\section{Data Extraction}

Data was extracted by one reviewer (PB) into a data extraction spreadsheet which was developed based on the Template for intervention description and replication (TIDieR) checklist. ${ }^{31}$ Data extraction was checked by a second reviewer (RS).

The following data were extracted: citation details; aims; total number of participants; number of groups; number in each group; number lost to attrition in each group; randomisation; blinding; time since stroke; selection criteria; measurement schedule; baseline arm function/score; frequency of MP sessions; duration of each MP session; the length of the entire MP intervention; number of completed sessions; total minutes of completed MP; duration and length of control intervention; baseline, post intervention and follow-up (where available) point estimates and measures of variability on outcome tools that measured activities or activity limitations. Where the manuscript did not present data, the authors were contacted for this information.

\section{Risk of bias (quality) assessment}

Two reviewers (RS and $\mathrm{KJ}$ ) independently assessed the quality of all included studies using the Physiotherapy Evidence Database (PEDro) ${ }^{36}$ criteria scores. Where possible, published assessments on the PEDro website were used to indicate the quality of included studies. In the absence of published scores, PEDro scores were independently assigned and then agreed by two reviewers (RS and KJ). 
193 Scores indicated poor (less than 2), moderate (3-5) or high quality (6-10) trials. ${ }^{36}$ Any discrepancies were resolved by discussion.

\section{Analysis}

Studies were synthesised narratively and, where possible, meta-analysis of the different continuous measures of upper-limb activity, presenting results as point estimates and $95 \%$ confidence intervals $(\mathrm{Cl})$ was also undertaken by one reviewer (AC). Funnel plots (plot of effect estimates from studies against a measure of precision) were used to judge risk of publication bias. Weighted mean differences (MD) were calculated where outcomes were measured on the same scale, with standardised weighted mean differences (SMDs) calculated where outcomes were measured on different scales for the same underlying construct. ${ }^{34}$ Random-effects models were estimated where SMDs were used to pool outcomes and fixed-effect models where MD were synthesised. Heterogeneity was assessed through visual inspection of forest plots and the calculation of the $X^{2}$ and $I^{2}$ statistics. Sub-group analyses explored the influence of time post stroke onset (using Stroke Recovery and Rehabilitation Roundtable, SRRR classification), ${ }^{37}$ severity of upper-limb involvement at baseline (i.e. Action Research Arm Test; ARAT 0-20; 21-40; 41-57) and the overall dose of mental practice delivered (minutes per day, calculated by dividing the total number of minutes of MP reported to be delivered by the total length of the MP intervention in days). This was categorised into low (below $25^{\text {th }}$ centile), medium (25-75 th centile), high doses (above $75^{\text {th }}$ centile).

Results were presented according to the Template for Intervention Description and Replication (TIDieR), ${ }^{31}$ and comprised consideration of who and when (including participant gender, the time since stroke using published criteria, ${ }^{37}$ the participants' 
217 cognitive function, and arm severity at baseline) and what and how much (the

218 viewpoint of MP, the simultaneous inclusion and the nature of other rehabilitative

219 interventions and the overall dose of MP provided).

\section{Results}

223 Initial searches yielded 1721 articles, which were reduced to 1239 after duplicates

224 were removed (see PRISMA diagram in Figure 1). After title, abstract, and then full

225 text screening, fifteen studies were selected for narrative review and presented in

226 Table 1. ${ }^{19,26,27,38-49}$ Four authors were contacted and asked to provide data which

227 would allow meta-analysis; ${ }^{44,46,47,49}$ one responded with data, another responded but

228 did not provide the data, two did not respond but the data for one of these two was

229 able to be extracted from Barclay-Goddard et al.'s (2011) Cochrane review. ${ }^{8}$ This left $^{2}$

23012 studies that were suitable for meta-analysis. ${ }^{19,26,27,38-46}$ The characteristics and

231 main findings of included studies are presented in Table 1.

232 Figure 1 here

233 Figure 1 PRISMA diagram to show article flow through the review

234 TABLE 1 here

235 Table 1 Characteristics of included studies 
238 PEDRO scores are displayed in Table 1. Seven studies were either of moderate ${ }^{19,26,38,39,44-46}$ or high quality $27,40-43,48,49$ whilst one was of poor quality. ${ }^{47}$

\section{Outcomes:}

Nine ${ }^{19,26,27,38-41,45,49}$ studies utilised the Action Research Arm Test to indicate upperlimb activity limitations as a primary or secondary outcome tool. The remaining six studies used either the Wolf Motor Function Test, ${ }^{42,48}$, Jebsen-Taylor hand test, ${ }^{43}$ Arm functional test - Functional Arm ability scale ${ }^{46}$ or Motor activity log. ${ }^{44,47}$ As these tools captured data predominantly at the level of activities they were collectively pooled for analysis. ${ }^{19,26,27,38-46}$ Meta-analysis of these 12 studies revealed the standardised weighted mean difference (SMD) for the overall effectiveness of MP upon measures of activity limitation (shown in Figure 2) was 0.6 (95\% confidence intervals, $\mathrm{Cl}: 0.32$ to $\left.0.88 ; n=328 ; 1^{2}=29 \%\right)$.

Figure 2 Forest plot to show the overall effectiveness of mental practice upon activity limitations of the upper-limb

\section{Who and when?}

256 Fourteen of the 15 included studies presented demographic data and reported the time since stroke (Table 1). ${ }^{19,26,27,38-46,48,50}$ There were more males than females (males: 282; females: 183) and participants were a mean of 59.2 (SD: 4.9) years old. Using standard criteria ${ }^{37}$, eight studies were conducted in the chronic period $26,38,39,42-$ 45,49 , two in the late sub-acute period ${ }^{27,41}$ with four being undertaken with people 
predominantly in the early sub-acute period after stroke. ${ }^{19,40,46,48}$ Meta-analysis of 12 studies showed that MP had the largest benefit upon activity limitations in the early subacute period (7 days to 3 months) after stroke (SMD:1.01, 95\% Cl: 0.53 to $1.5 ; 3$ studies, $\left.\mathrm{n}=76 ; \mathrm{I}^{2}=0 \%\right)^{19,40,46}$ followed by the chronic period ( 6 months and later; SMD: 0.65, 95\% Cl: 0.32 to $0.99 ; 7$ studies, $\left.n=151 \mathrm{I}^{2}=0 \%\right)^{13,26,38,39,42-44}$ (Figure 3). Changes in activity limitation after MP during the late sub-acute period ( 3 to 6 months) were small and non-significant (SMD:0.09, 95\% Cl: -0.3 to $0.48, \mathrm{p}=0.65 ; 2$ studies, $\mathrm{n}=111$ $\left.\mathrm{I}^{2}=0 \%\right)^{27,41}$

FIGURE 3 HERE

Figure 3 Forest plot to show subgroup analysis (fixed effects) of time after stroke and effectiveness of mental practice upon upper limb activities

All studies required participants to have no or very mild cognitive deficits in order to take part. Ten from 15 studies screened people for cognitive dysfunction prior to mental status questionnaire, ${ }^{27}$ whilst another used the Wechsler Memory scale. ${ }^{41}$

For those eight studies $(n=226)$ that reported baseline arm function using the ARAT, ${ }^{19,26,27,38-41,45}$ most included participants who had moderate arm limitations (median ARAT score: 25 range: 5-49). Only one study included participants who would be classed as having severe arm limitations on the ARAT (mean ARAT score: $5)^{41}$. As presented in Figure 4, meta-analysis showed that MP had the greatest benefit for those with the most severe upper-limb limitations (ARAT scores of 0-20; weighted mean difference, WMD: $7.33,95 \% \mathrm{Cl}: 0.94$ to13.72; 3 studies, ${ }^{39-41} \mathrm{n}=82$; 
$285 \mathrm{I}^{2}=0 \%$ ) followed by those with moderate limitations (ARAT scores from 21-40; WMD: effective in improving limitations in those with the most mild upper-limb involvement, although this was only based on one study (ARAT scores from 41-57; WMD: 2.50, $95 \% \mathrm{Cl}:-4.38$ to $9.38, p=0.48 ; 1$ study, $\left.{ }^{45} \mathrm{n}=29\right)$.

FIGURE 4 HERE

291

Figure 4 Forest plot to show subgroup analysis (random effects) of the effects of initial arm severity, measured using the ARAT, upon the effectiveness of mental practice

\section{What and how much?}

Ten studies did not clearly specify which perspective (first or third person) was used during MP. Of the five studies that did, four solely utilised a first person perspective $\mathrm{e}^{19,39,41,48}$ whilst one used both first and third person. ${ }^{43}$

In ten of the studies, MP was delivered in addition to conventional therapy/other usual rehabilitation ${ }^{13,19,26,38-40,42,43,46,49}$ however there was little included detail of what this comprised.

The mean length of the MP intervention was 4.7 weeks (SD: 1.9) with a median of 3 sessions (range 2-15) being provided each week. One study compared three different durations of MP intervention so was excluded from this analysis. ${ }^{50}$ The mean duration of a typical MP session in the other 14 studies was 28.4 minutes (SD: 15.1)..$^{19,26,27,38-48}$ Dose was calculated to indicate the average amount of MP received per day (total number of minutes of MP divided by the total length of the intervention 
in days). The mean average dose was 20.3 minutes/day (SD: 14) from the 14 studies that used a single MP intervention ${ }^{19,26,27,38-48}$ (Table 1).

310 For meta-analysis, the data were split into low, medium and high doses using the

311 method of calculation described earlier. Two studies used a low dose of MP $(\leq 6.6$

312 minutes/day), ${ }^{26,43}$ five used a medium dose ( 6.7 to 32 minutes/day $)^{13,38,39,42,44}$ whilst

313 five used a high dose ( $\geq 32.1$ minutes/day). ${ }^{19,27,40,41,46}$ As shown in Figure 5, a lower

314 dose appeared to confer somewhat greater benefit to upper-limb function (SMD:

$3150.89,95 \% \mathrm{Cl}: 0.04$ to $1.74 ; 2$ studies, ${ }^{26,43} \mathrm{n}=25 ; \mathrm{I}^{2}=0 \%$ ) than a medium (SMD: 0.61 , $31695 \%$ : 0.25 to $0.98 ; 5$ studies, ${ }^{13,38,39,42,44} \mathrm{n}=126 \mathrm{I}^{2}=0 \%$ ) or high dose (SMD: $0.57,95 \%$

317 Cl: 0.05 to $1.08 ; 5$ studies, $\left.{ }^{19,27,40,41,46} \mathrm{n}=177 ;\left.\right|^{2}=60 \%\right)$.

319 Figure 5 Forest plot to show subgroup analysis of the effects of dose upon activity 320 limitations

Six of the 15 studies provided control treatments to match the time and attention given the intervention group. ${ }^{19,27,38,43,44,48}$ They provided additional conventional therapy, ${ }^{19,44}$ relaxation recordings, ${ }^{38,43}$ additional treatment based on the neurodevelopmental technique ${ }^{48}$ or visual imagery training. ${ }^{27}$ The remaining nine studies did not detail the provision of additional control treatment.

\section{Discussion}

329 This systematic review evaluates the effectiveness of MP for the upper-limb after stroke. It aimed to determine in whom and when after stroke it might have benefit 
and to identify the dose of MP that might have the greatest effect. The main results of this review were based on largely moderate to high quality trials and indicate that MP can confer significant reductions in upper-limb activity limitations. Heterogeneity in the meta-analyses were low (less than $29 \%$ ) supporting the validity of these results.

An important finding is that the magnitude of reported benefit of MP on upper-limb activity limitations (SMD: 0.60, 95\% confidence intervals, $\mathrm{Cl}$ : 0.32 to $0.88 ; 12$ studies, $\mathrm{n}=328$ ) exceeds that reported for other recognised upper-limb treatments in comparable studies of people after stroke. These include repetitive task training (SMD: $0.25,95 \% \mathrm{Cl}$ : 0.01 to $0.49 ; 11$ studies $n=749)^{24}$ and constraint induced movement therapy (SMD: $0.24,95 \% \mathrm{Cl}:-0.05$ to $0.52 ; 42$ studies, $n=1453)^{51}$.

Despite the apparent superiority of MP to other upper-limb interventions, MP is reported to be used much less frequently than either repetitive task training or constraint induced movement therapy in clinical practice. ${ }^{32}$ This indicates that further work to support the implementation of MP into routine therapy practice is clearly warranted.

The results of the current study are similar to that of the most recent Cochrane review of MP for the upper-limb which reported analogous effect sizes of the overall effectiveness of MP from 15 studies (SMD: $0.66,95 \% \mathrm{Cl}: 0.39$ to $0.94 ; n=397) .{ }^{9}$ However, a larger analysis reported a smaller effect (SMD: 0.36, 95\% Cl: 0.16 to $0.55 ; 18$ studies, $n=644) .{ }^{28}$ The disparity between these two reviews may be attributable to differences in the number of studies and participants included and the analytical approach; Guerra et al. (2017) ${ }^{28}$ pooled data from studies measuring both impairment and activity limitations, whilst Barclay-Goddard et al. (2020) did not. ${ }^{9}$ The findings could indicate a trend for lower effectiveness of MP on impairments in 
comparison to activity limitations. However, this supposition is not supported in the analysis of impairment outcomes by Barclay-Goddard et al. (2020) (SMD: 0.59, $95 \% \mathrm{Cl}: 0.30-0.87 ; 15$ studies, $n=397)^{9}$. This warrants further investigation.

\section{Who? Patient selection and time since stroke}

All trials selected participants that had normal or only mild cognitive dysfunction after stroke. The effect of reduced cognition upon the ability to undertake MP after stroke remains uncertain. Several studies have shown that mental imagery after stroke may take longer to complete when compared to healthy and/or younger controls $20,21,52,53$ but no studies have provided explicit evidence of the minimal cognitive function required to successfully complete MP. Future studies should therefore consider broadening inclusion criteria to incorporate sub-groups of those after stroke with moderate cognitive deficits to determine if they may benefit from MP.

None of the included trials stratified participants at baseline. As well as potentially attenuating the estimates of effectiveness, the absence of stratification leads to difficulty in knowing the optimal time after stroke and the severity of upper-limb limitations that are likely to benefit most from MP. However, the sub-analyses presented in this review suggest that MP delivered in the early subacute and chronic phases after stroke and to those with the most severe arm deficits (scoring 0-20 on the ARAT) may gain the most from MP.

Whilst others have found small differences between the effectiveness of MP provided in the first six months after stroke or later $^{9}$ (less than 6 months: SMD: 0.48, 95\% Cl: -0.04 to $0.99 ; 5$ studies, $n=188$; $\geq$ more than 6 months: SMD: $0.75,95 \% \mathrm{Cl}$ : 0.44 to $1.06,8$ studies, $n=179),{ }^{9}$ our use of the SRRR criteria allowed more detailed consideration of time periods. This revealed that the early subacute (seven days to 
three months after stroke) group had the largest change in activity limitations after using MP. The larger magnitude of changes during this early period is perhaps unsurprising as the most rapid and the majority of endogenous plasticity, and thus recovery of motor control, is typically observed in the first few weeks after stroke. ${ }^{37,54}$ However, in line with the Barclay et al. (2020) review, ${ }^{9}$, a smaller but significant benefit was also seen in people at least 6 months after stroke, suggesting that MP may have different mechanisms of effect depending upon when after stroke it is used. Collectively these results suggest that MP can improve upper-limb function at multiple time points after stroke, and that work to understand the mechanism, and potential differences in mechanisms, depending upon the time period in which it is applied after stroke is warranted.

Our finding that those with the most severe deficits exhibited substantial and significant benefit from MP is novel and is particularly noteworthy as this benefit (MD: 7.3, $95 \% \mathrm{Cl}: 0.94$ to $13.7, \mathrm{I}^{2}=0 \%$ ) exceeds the minimal clinically important difference for the ARAT (5.7). ${ }^{55}$ No other reviews of MP for the upper-limb have considered the severity of upper-limb deficits upon the effectiveness of MP. ${ }^{9,28,56}$ Our finding indicates that MP could provide a promising treatment for people with severe upperlimb limitations, who typically cannot independently participate in other recognised treatments (such as repetitive task training), as they have little voluntary movement. The strength of this conclusion is limited by the wide confidence intervals, relatively small number of studies in each subgroup, although heterogeneity was low, and because the cut offs used in this analysis were arbitrarily assigned (ARAT scores: severe: 0-20; moderate: 21-40; mild:41-57) to allow comparison. However, we chose not to use more widely recognised ARAT cut off scores (severe: 0 -10; moderate: 1156; mild: 57$)^{55}$ as this would mean all but one study ${ }^{41}$ would be considered to have 
moderate limitations and so any subtleties in the response to MP would be missed. Whilst analysing the severity of upper-limb limitations as we did is not standard, it highlights that the effect of MP upon severe activity limitations after stroke is worthy of further study in this group.

\section{What and How much? Delivery and dose of MP after stroke}

In this review there was little indication to determine which perspective (first or third person) used during MP was superior as most studies did not indicate the perspective used. Mental imagery from a first person (egocentric) perspective is generally agreed to be more effective than from a third person perspective and so is more widely used in published research protocols ${ }^{7}$ but there is little empirical evidence to support this. ${ }^{11,43}$ Few studies also indicated if or how MP training was supervised. This is important as how MP is provided will have important time and cost implications for therapy services, significantly influencing cost effectiveness. Lack of detail regarding how MP is provided is a common criticism of studies reporting MP interventions. ${ }^{57}$ This could be remedied by adopting recognised frameworks to deliver MP used in sport (e.g. Physical, Environment, Task, Timing, Learning, Emotion and Perspective, PETTLEP ${ }^{11}$ and by the assiduous use of intervention reporting guidelines in future studies (e.g. TIDieR). ${ }^{31,57}$

Interestingly, all but one ${ }^{41}$ of the 15 included studies in this review delivered MP as a single massed practice session on each day it was delivered. This contrasts with the superiority of distributed over massed practice seen in motor learning ${ }^{58}$ and the findings of a small study in which distributed MP (20 minutes, three times a day, $n=13$ ) produced significantly larger gains in upper-limb recovery after stroke when compared to once daily therapy for 60 minutes $(n=14) .{ }^{59}$ This suggests that future 
studies should consider delivering shorter but more frequent MP sessions to elicit greater gains in function.

431 The meta-analysis of the dose delivered in this review indicated that a low or medium dose (low: less than 6.6 minutes per day medium: more than $>6.7$ to less than 32.1 minutes/day) appeared slightly more beneficial than higher doses of MP (more than32.2 minutes). If accurate, this indicates that MP could provide an effective intervention without requiring substantial increases in therapist time and costs. However, this is perhaps unlikely as these findings contradict the accepted linear relationship between upper-limb therapy dose and response, ${ }^{60}$ and instead could be explained by the doses of MP delivered in all included studies being below the amount needed to elicit optimal benefit. Other studies indicate that therapy for the upper-limb must be delivered intensively in order to show an optimal benefit, 61,62 which is likely to comprise several hours of intensive daily treatment. ${ }^{60,63}$ In studies included in this review, one hour was the maximum daily amount of MP delivered and this was only delivered in two trials. ${ }^{41,46}$ Others have shown no significant differences in outcomes between MP delivered for an hour a day when compared to lower doses 20 and 40 minutes/day. ${ }^{49}$ This suggests that future trials should compare doses of a few minutes of MP to much more intensive practice akin to that 447 in studies of upper limb rehabilitation that have shown significant benefit. It is also 448 important to note that, both in this study and others, judgements of dose and intensity were estimated solely from the duration that MP was provided. Detailing the numbers of repetitions and the joints and movements targeted of mentally practiced movements provided by the MP script would provide a more accurate estimation of

452 the intensity of training and should be reported in future studies, although it is 
recognised that an individual's adherence to imagining movements cannot be measured.

\section{Limitations}

456 Funnel plots suggest that the findings of this systematic review may be skewed by publication bias, with asymmetrical plots suggesting a lack of small studies showing no benefit from the comparator interventions. Inevitably this can lead to overestimation of the effectiveness of MP. Potential bias in the judgements of which studies were included in the review may also skew results. Whilst data extraction was checked, it was undertaken by one reviewer which may have introduced error. Its wider validity is also restricted by the inclusion of only full-text articles available in English and exclusion of articles that did not measure changes in activity limitations of the upper-limb. The exclusion of studies that measured impairment was primarily because a reduction in activity limitations is recognised to be more meaningful to people after stroke than alterations in impairment. ${ }^{64}$ Taken alongside the knowledge that finding ways to effectively rehabilitate the upper-limb after stroke is a recognised priority for both stroke survivors and clinicians ${ }^{30}$ the focus upon activity limitations in this review increases its clinical validity and ultimately its usefulness to clinicians and people after stroke.

Interestingly, the studies included in this review did not always reflect the 'typical' person who has had a stroke which limits the broader generalisability of the findings. Included studies had relatively young participants with a mean age of 59 (SD: 5) years and preferentially recruited males (there were almost 100 more males than 475 females). In Europe and Australasia, the average age for first stroke is markedly older (around 70 years), stroke is more common in women than men, ${ }^{65}$ and findings 
477 between sexes are not directly transferable as females tend to have poorer

478 functional recovery. ${ }^{66}$

479 A further limitation to the findings of this review is that no studies comprehensively 480 examined compliance and fidelity to the MP intervention; others have reported low 481 patient and therapist compliance to MP, ${ }^{67} \mathrm{MP}$ interventions are often not clearly defined ${ }^{57}$ and few therapists report using MP as part of therapy for the upper-limb after stroke ${ }^{32}$ suggesting that the training and practical requirements of implementing MP need to be considered alongside its clinical effectiveness.

\section{Conclusions}

487 The results of this systematic review and meta-analysis indicated that MP can significantly improve activity limitations of the upper-limb after stroke and that it appears more effective than several other, more frequently used interventions for the upper-limb. This highlights that work is warranted to explore and support the successful implementation of MP into clinical practice so that more people can benefit from using it as part of their rehabilitation after stroke. The finding that MP provides significant and substantial benefit that markedly exceeds the minimal clinically important difference for the ARAT in those people after stroke with the most severe limitations of the upper-limb is particularly novel and suggests that MP may constitute a promising therapy for this subgroup.

497 Future trials should seek to stratify people based on the severity of upper-limb function and/or their potential for recovery of the upper-limb to aid understanding of who may benefit the most from MP. Further work is also needed to standardise the delivery of MP; including identifying an optimal dose, standardising exactly how MP 
501 is being used (first or third person viewpoints) and the number of repetitions of the 502 included movements in the MP intervention. This could be done by use of a detailed 503 intervention reporting too ${ }^{31}$ and established MP intervention structure. ${ }^{11}$ 
506

507

508

509

510

511

512

513

514

515

516

517

518

519

520

521

522

523

524

525

526

527

528

529

530

531

532

533

534

535

536

537

538

539

540

541

542

1. Feigin $\mathrm{VL}$, Forouzanfar $\mathrm{MH}$, Krishnamurthi R, et al. Global and regional burden of stroke during 1990-2010: findings from the Global Burden of Disease Study 2010. Lancet.

2014;383(9913):245-254.

2. Broeks JG, Lankhorst GJ, Rumping K, Prevo AJ. The long-term outcome of arm function after stroke: results of a follow-up study. Disabil Rehabil. 1999;21(8):357-364.

3. Chen CM, Tsai CC, Chung CY, Chen CL, Wu KP, Chen HC. Potential predictors for health-related quality of life in stroke patients undergoing inpatient rehabilitation. Health and quality of life outcomes. 2015;13:118. doi:10.1186/s12955-015-0314-5

4. Lieshout ECC van, van de Port IG, Dijkhuizen RM, Visser-Meily JMA. Does upper limb strength play a prominent role in health-related quality of life in stroke patients discharged from inpatient rehabilitation? Topics in Stroke Rehabilitation. Published online March 9, 2020:1-9. doi:10.1080/10749357.2020.1738662

5. Royal College of Physicians. National Clinical Guideline for Stroke.; 2016.

6. Hebert $D$, Lindsay MP, McIntyre A, et al. Canadian stroke best practice recommendations: Stroke rehabilitation practice guidelines, update 2015. International Journal of Stroke. 2016;11(4):459-484. doi:10.1177/1747493016643553

7. Moran A, Guillot A, Maclntyre T, Collet C. Re-imagining motor imagery: Building bridges between cognitive neuroscience and sport psychology. British Journal of Psychology. 2012;103:224-247. doi:10.1111/j.2044-8295.2011.02068.x

8. Barclay-Goddard RE, Stevenson TJ, Poluha W, Thalman L. Mental practice for treating upper extremity deficits in individuals with hemiparesis after stroke. Cochrane Database of Systematic Reviews. Published online 2011:N.PAG-N.PAG.

9. Barclay RE, Stevenson TJ, Poluha W, Semenko B, Schubert J. Mental practice for treating upper extremity deficits in individuals with hemiparesis after stroke. Cochrane Stroke Group, ed. Cochrane Database of Systematic Reviews. Published online May 25, 2020. doi:10.1002/14651858.CD005950.pub5

10. de Vries S, Tepper M, Feenstra W, Oosterveld H, Boonstra AM, Otten B. Motor imagery ability in stroke patients: the relationship between implicit and explicit motor imagery measures. Front Hum Neurosci. 2013;7:790. doi:https://dx.doi.org/10.3389/fnhum.2013.00790

11. Wakefield C, Smith D. Perfecting Practice: Applying the PETTLEP Model of Motor Imagery. Journal of Sport Psychology in Action. 2012;3(1):1-11. doi:10.1080/21520704.2011.639853

12. Schuster $\mathrm{C}$, Hilfiker $\mathrm{R}, \mathrm{Amft} \mathrm{O}$, et al. Best practice for motor imagery: a systematic literature review on motor imagery training elements in five different disciplines. BMC Medicine. 2011;9(1):75. doi:10.1186/1741-7015-9-75

13. Park $\mathrm{CH}$, Chang $\mathrm{WH}$, Lee $\mathrm{M}$, et al. Predicting the performance of motor imagery in stroke patients: multivariate pattern analysis of functional MRI data. Neurorehabil Neural Repair. 2015;29(3):247-254. doi:https://dx.doi.org/10.1177/1545968314543308 
14. O'Shea H, Moran A. Does Motor Simulation Theory Explain the Cognitive Mechanisms Underlying Motor Imagery? A Critical Review. Front Hum Neurosci. 2017;11. doi:10.3389/fnhum.2017.00072

15. Jeannerod M, Decety J. Mental motor imagery: a window into the representational stages of action. Current Opinion in Neurobiology. 1995;5(6):727-732. doi:10.1016/0959-4388(95)800999

16. Braun N, Kranczioch C, Liepert J, et al. Motor Imagery Impairment in Postacute Stroke Patients. Neural Plast. Published online 2017:13. doi:10.1155/2017/4653256

17. Sharma N, Pomeroy VM, Baron J-C. Motor Imagery: A Backdoor to the Motor System After Stroke? Stroke. 2006;37(7):1941-1952. doi:10.1161/01.STR.0000226902.43357.fc

18. Butler AJ, Page SJ. Mental practice with motor imagery: evidence for motor recovery and cortical reorganization after stroke. Archives of Physical Medicine \& Rehabilitation. 2006;87:S211.

19. Li F, Zhang T, Li BJ, Zhang W, Zhao J, Song LP. Motor imagery training induces changes in brain neural networks in stroke patients. Nerual Regen. 2018;13(10):1771-1781. doi:https://dx.doi.org/10.4103/1673-5374.238616

20. de Vries S, Tepper M, Otten B, Mulder T. Recovery of motor imagery ability in stroke patients. rehabil. 2011;2011:283840. doi:https://dx.doi.org/10.1155/2011/283840

21. Santoro S, Lo Buono V, Corallo F, et al. Motor imagery in stroke patients: a descriptive review on a multidimensional ability. International Journal of Neuroscience. 2019;129(8):821-832. doi:10.1080/00207454.2019.1567509

22. Storm V, Utesch T. Mental Practice Ability Among Stroke Survivors: Investigation of Gender and Age. Front Psychol. 2019;10:1568. doi:10.3389/fpsyg.2019.01568

23. Wolf SL, Thompson PA, Winstein CJ, et al. The EXCITE stroke trial: comparing early and delayed constraint-induced movement therapy. Stroke. 2010;41(10):2309-2315. doi:10.1161/STROKEAHA.110.588723

24. French $\mathrm{B}$, Thomas $\mathrm{LH}$, Coupe J, et al. Repetitive task training for improving functional ability after stroke. Cochrane Database Syst Rev. 2016;11:CD006073. doi:10.1002/14651858.CD006073.pub3

25. Page SJ. Imagery improves upper extremity motor function in chronic stroke patients: a pilot study. Occupational Therapy Journal of Research. 2000;20(3):200-215.

26. Page $S J$, Levine $P$, Sisto $S A$, Johnston MV. Mental practice combined with physical practice for upper-limb motor deficit in subacute stroke. Physical therapy. 2001;81(8):1455-1462.

27. letswaart $\mathrm{M}$, Johnston $\mathrm{M}$, Dijkerman $\mathrm{HC}$, et al. Mental practice with motor imagery in stroke recovery: randomized controlled trial of efficacy. Brain. 2011;134(Pt 5):1373-1386. doi:https://dx.doi.org/10.1093/brain/awr077

28. Guerra ZF, Lucchetti ALG, Lucchetti G. Motor Imagery Training After Stroke: A Systematic Review and Meta-analysis of Randomized Controlled Trials. J Neurol Phys Ther. 2017;41(4):205214. doi:https://dx.doi.org/10.1097/NPT.0000000000000200 
29. Carrasco DG, Cantalapiedra JA. Effectiveness of motor imagery or mental practice in functional recovery after stroke: a systematic review. Neurologia. 2016;31(1):43-52. doi:10.1016/j.nrl.2013.02.003

30. Pollock A, St George B, Fenton M, Firkins L. Top 10 research priorities relating to life after stroke--consensus from stroke survivors, caregivers, and health professionals. International journal of stroke : official journal of the International Stroke Society. 2014;9(3):313-320. doi:10.1111/j.1747-4949.2012.00942.x

31. Hoffmann TC, Glasziou PP, Boutron I, et al. Better reporting of interventions: template for intervention description and replication (TIDieR) checklist and guide. BMJ. 2014;348:g1687. doi:10.1136/bmj.g1687

32. Stockley R, Peel R, Jarvis K, Connell L. Current therapy for the upper limb after stroke: a crosssectional survey of UK therapists. BMJ Open. 2019;9(9). doi:10.1136/bmjopen-2019-030262

33. Moher D, Liberati A, Tetzlaff J, Altman DG. Preferred Reporting Items for Systematic Reviews and Meta-Analyses: The PRISMA Statement. PLoS Med. 2009;6(7). doi:10.1371/journal.pmed.1000097

34. Higgins J, Thomas J. Cochrane Handbook for Systematic Reviews of Interventions Version 5.1.0 [Updated March 2011]. 6th ed. The Cochrane Collaboration.; 2019.

35. World Health O. International Classification of Functioning, Disability and Health (ICF). WHO; 2001.

36. de Morton NA. The PEDro scale is a valid measure of the methodological quality of clinical trials: a demographic study. Aust J Physiother. 2009;55(2):129-133.

37. Bernhardt J, Hayward KS, Kwakkel G, et al. Agreed Definitions and a Shared Vision for New Standards in Stroke Recovery Research: The Stroke Recovery and Rehabilitation Roundtable Taskforce. Neurorehabil Neural Repair. 2017;31(9):793-799. doi:10.1177/1545968317732668

38. Page SJ, Levine $P$, Leonard AC. Effects of mental practice on affected limb use and function in chronic stroke. Archives of Physical Medicine and Rehabilitation. 2005;86(3):399-402. doi:10.1016/j.apmr.2004.10.002

39. Page SJ, Levine $P$, Leonard A, Page SJ, Levine $P$, Leonard A. Mental practice in chronic stroke: results of a randomized, placebo-controlled trial. Stroke (00392499). 2007;38(4):1293-1297.

40. Liu H, Song LP, Zhang T. Mental practice combined with physical practice to enhance hand recovery in stroke patients. Behav. 2014;2014:876416. doi:https://dx.doi.org/10.1155/2014/876416

41. Welfringer A, Leifert-Fiebach G, Babinsky R, Brandt T. Visuomotor imagery as a new tool in the rehabilitation of neglect: a randomised controlled study of feasibility and efficacy. Disabil Rehabil. 2011;33(21-22):2033-2043. doi:https://dx.doi.org/10.3109/09638288.2011.556208

42. Seong-Sik K, Byoung-Hee L. Motor imagery training improves upper extremity performance in stroke patients. Journal of Physical Therapy Science. 2015;27(7):2289-2291.

43. Nilsen DM. Does view matter? the effect of imagery perspective on occupational performance in individuals' post stroke. Published online 2012. 
44. Nayeem Z, Majumi M, Fuzail A. Effect of Mental Imagery on Upper Extremity Function in Stroke Patients. Indian Journal of Physiotherapy \& Occupational Therapy. 2012;6(1):88-90.

45. Park, J., Lee, N, Cho M, Kim D, Yang Y. Effects of mental practice on stroke patients' upper extremity function and daily activity performance. Journal of Physical Therapy Science. 2015;27(4):1075-1077. doi:10.1589/jpts.27.1075

46. Riccio I, lolascon G, Barillari MR, Gimigliano R, Gimigliano F. Mental practice is effective in upper limb recovery after stroke: a randomized single-blind cross-over study. Eur J Phys Rehabil Med. 2010;46(1):19-25.

47. Kanwar, S., Kashoo FZ. Comparing the Immediate effective of auditory and visual mental imagery on reaching and grasping task amony sub acute stroke patients. Indian journal of Physiotherapy and Occupational Therapy. 2011;5(2):162-166.

48. Timmermans AA, Verbunt JA, van Woerden R, Moennekens M, Pernot DH, Seelen HA. Effect of mental practice on the improvement of function and daily activity performance of the upper extremity in patients with subacute stroke: a randomized clinical trial. J Am Med Dir Assoc. 2013;14(3):204-212. doi:https://dx.doi.org/10.1016/j.jamda.2012.10.010

49. Page SJ, Dunning K, Hermann V, Leonard A, Levine P. Longer versus shorter mental practice sessions for affected upper extremity movement after stroke: a randomized controlled trial. Clin Rehabil. 2011;25(7):627-637. doi:https://dx.doi.org/10.1177/0269215510395793

50. Page SJ, Murray C, Hermann V, Levine P. Retention of Motor Changes in Chronic Stroke Survivors Who Were Administered Mental Practice. Archives of Physical Medicine \& Rehabilitation. 2011;92(11):1741-1745. doi:10.1016/j.apmr.2011.06.009

51. Corbetta D, Sirtori V, Castellini G, Moja L, Gatti R. Constraint-induced movement therapy for upper extremities in people with stroke. Cochrane Database of Systematic Reviews. 2015;(10):CD004433. doi:https://dx.doi.org/10.1002/14651858.CD004433.pub3

52. Geiger M, Bonnyaud C, Fery Y-A, Bussel B, Roche N. Evaluating the Effect of Cognitive Dysfunction on Mental Imagery in Patients with Stroke Using Temporal Congruence and the Imagined 'Timed Up and Go' Test (iTUG). PLoS One. 2017;12(1). doi:10.1371/journal.pone.0170400

53. Malouin F, Richards CL, Durand A. Slowing of Motor Imagery after a Right Hemispheric Stroke. Stroke Res Treat. 2012;2012. doi:10.1155/2012/297217

54. Cortes JC, Goldsmith J, Harran MD, et al. A Short and Distinct Time Window for Recovery of Arm Motor Control Early After Stroke Revealed With a Global Measure of Trajectory Kinematics. Neurorehabilitation and neural repair. 2017;31(6):552-560. doi:https://dx.doi.org/10.1177/1545968317697034

55. van der Lee JH, Beckerman $\mathrm{H}$, Lankhorst GJ, Bouter LM. The responsiveness of the Action Research Arm test and the Fugl-Meyer Assessment scale in chronic stroke patients. Journal of rehabilitation medicine. 2001;33(3):110-113.

56. Kho AY, Liu KP, Chung RC. Meta-analysis on the effect of mental imagery on motor recovery of the hemiplegic upper extremity function. Aust Occup Ther J. 2014;61(2):38-48. doi:https://dx.doi.org/10.1111/1440-1630.12084 
57. Harris JE, Hebert A. Utilization of motor imagery in upper limb rehabilitation: a systematic scoping review. Clin Rehabil. 2015;29(11):1092-1107. doi:https://dx.doi.org/10.1177/0269215514566248

58. Lee TD, Genovese ED. Distribution of Practice in Motor Skill Acquisition: Learning and Performance Effects Reconsidered. Research Quarterly for Exercise and Sport. 1988;59(4):277287. doi:10.1080/02701367.1988.10609373

59. Page SJ, Hade EM, Pang J. Retention of the spacing effect with mental practice in hemiparetic stroke. Exp Brain Res. 2016;234(10):2841-2847. doi:https://dx.doi.org/10.1007/s00221-0164686-5

60. Schneider EJ, Lannin NA, Ada L, Schmidt J. Increasing the amount of usual rehabilitation improves activity after stroke: a systematic review. J Physiother. 2016;62(4):182-187. doi:10.1016/j.jphys.2016.08.006

61. McCabe J, Monkiewicz M, Holcomb J, Pundik S, Daly JJ. Comparison of robotics, functional electrical stimulation, and motor learning methods for treatment of persistent upper extremity dysfunction after stroke: a randomized controlled trial. Arch Phys Med Rehabil. 2015;96(6):981-990. doi:10.1016/j.apmr.2014.10.022

62. Daly JJ, McCabe JP, Holcomb J, Monkiewicz M, Gansen J, Pundik S. Long-Dose Intensive Therapy Is Necessary for Strong, Clinically Significant, Upper Limb Functional Gains and Retained Gains in Severe/Moderate Chronic Stroke. Neurorehabil Neural Repair. 2019;33(7):523-537. doi:10.1177/1545968319846120

63. Borschmann K, Hayward KS, Raffelt A, Churilov L, Kramer S, Bernhardt J. Rationale for Intervention and Dose Is Lacking in Stroke Recovery Trials: A Systematic Review. Stroke Res Treat. 2018;2018. doi:10.1155/2018/8087372

64. Duncan Millar J, van Wijck F, Pollock A, Ali M. Outcome measures in post-stroke arm rehabilitation trials: do existing measures capture outcomes that are important to stroke survivors, carers, and clinicians? Clin Rehabil. 2019;33(4):737-749. doi:10.1177/0269215518823248

65. Phan HT, Blizzard CL, Reeves MJ, et al. Sex Differences in Long-Term Quality of Life Among Survivors After Stroke in the INSTRUCT. Stroke. 2019;50(9):2299-2306. doi:10.1161/STROKEAHA.118.024437

66. Gall SL, Tran PL, Martin K, Blizzard L, Srikanth V. Sex Differences in Long-Term Outcomes After Stroke: Functional Outcomes, Handicap, and Quality of Life. Stroke. 2012;43(7):1982-1987. doi:10.1161/STROKEAHA.111.632547

67. Bovend'Eerdt TJ, Dawes H, Sackley C, Izadi H, Wade DT. An Integrated Motor Imagery Program to Improve Functional Task Performance in Neurorehabilitation: A Single-Blind Randomized Controlled Trial. Archives of Physical Medicine and Rehabilitation. 2010;91(6):939-946. doi:10.1016/j.apmr.2010.03.008

\section{Figure 1 NO LEGEND}

Figure 2 
702

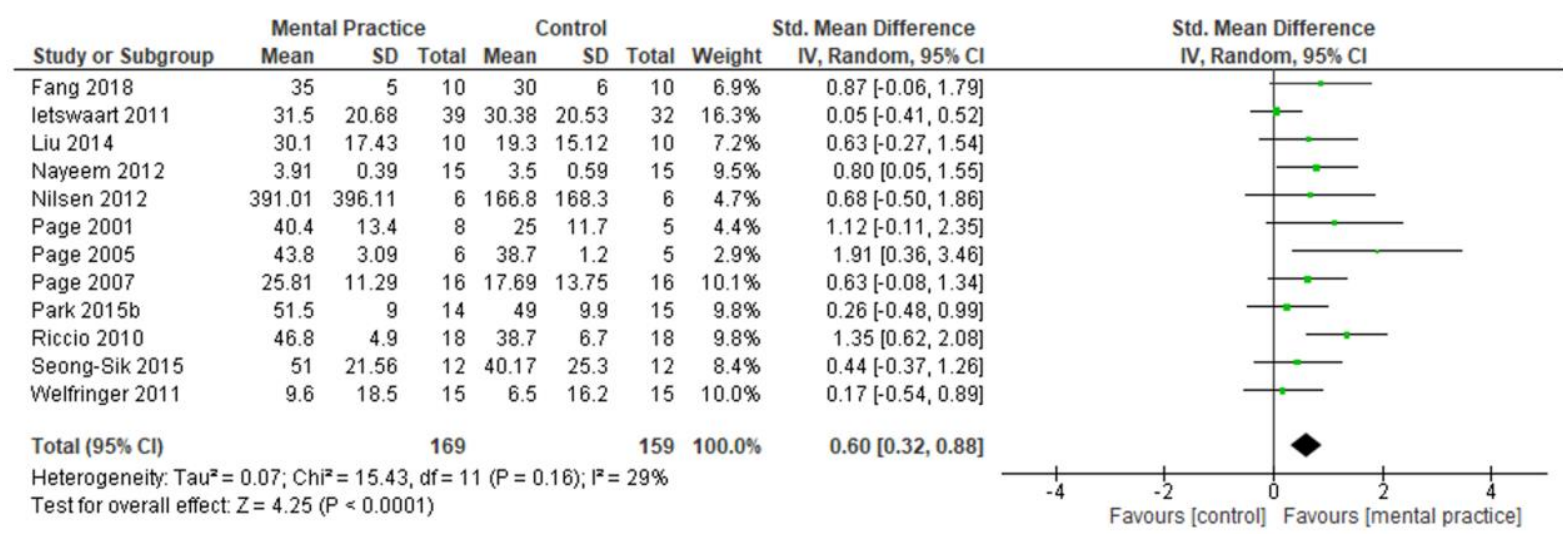

Figure 3

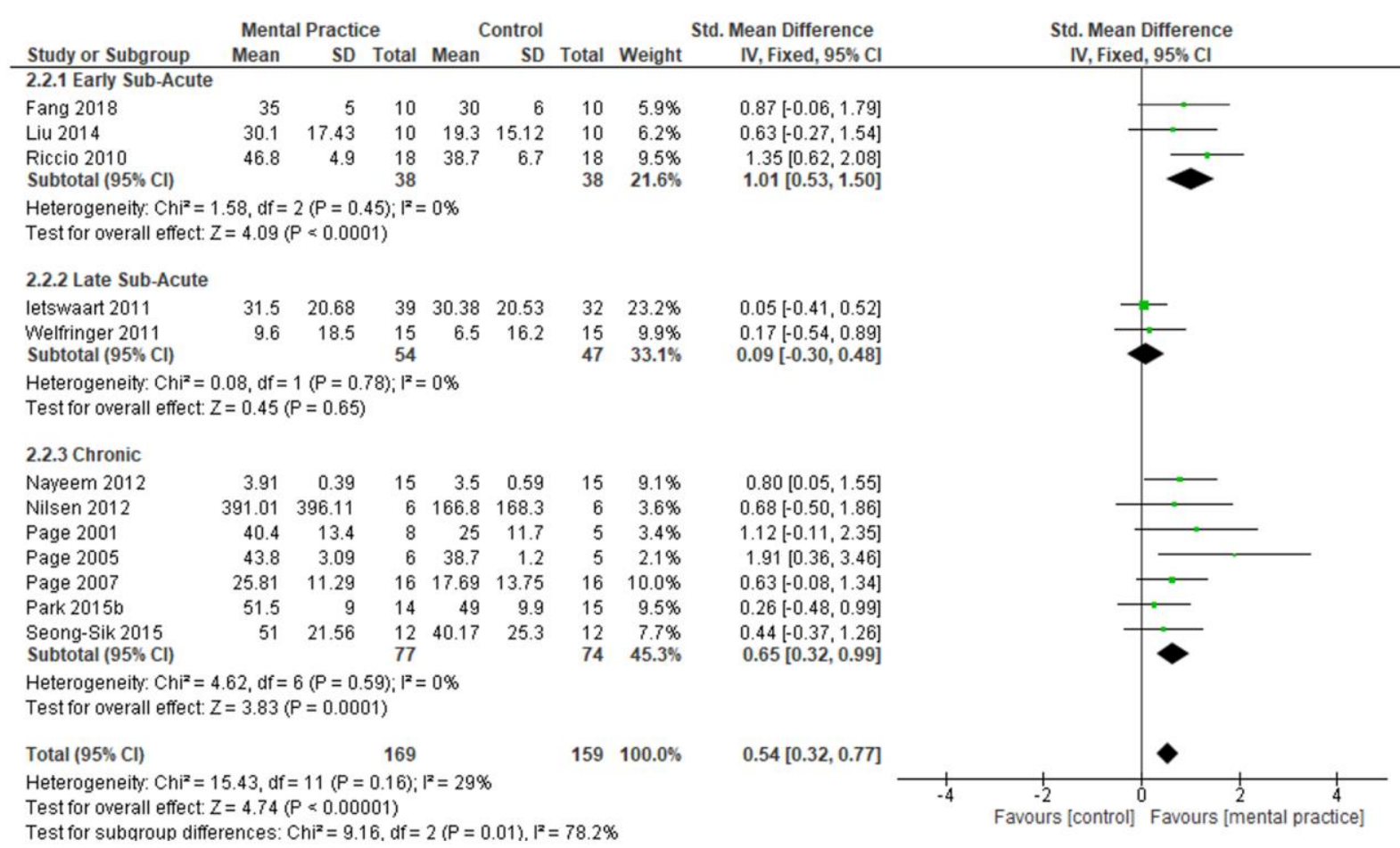

709 Legend: Data in Nilsen et al (2012) are reversed so that improvement is indicated by a higher score. 


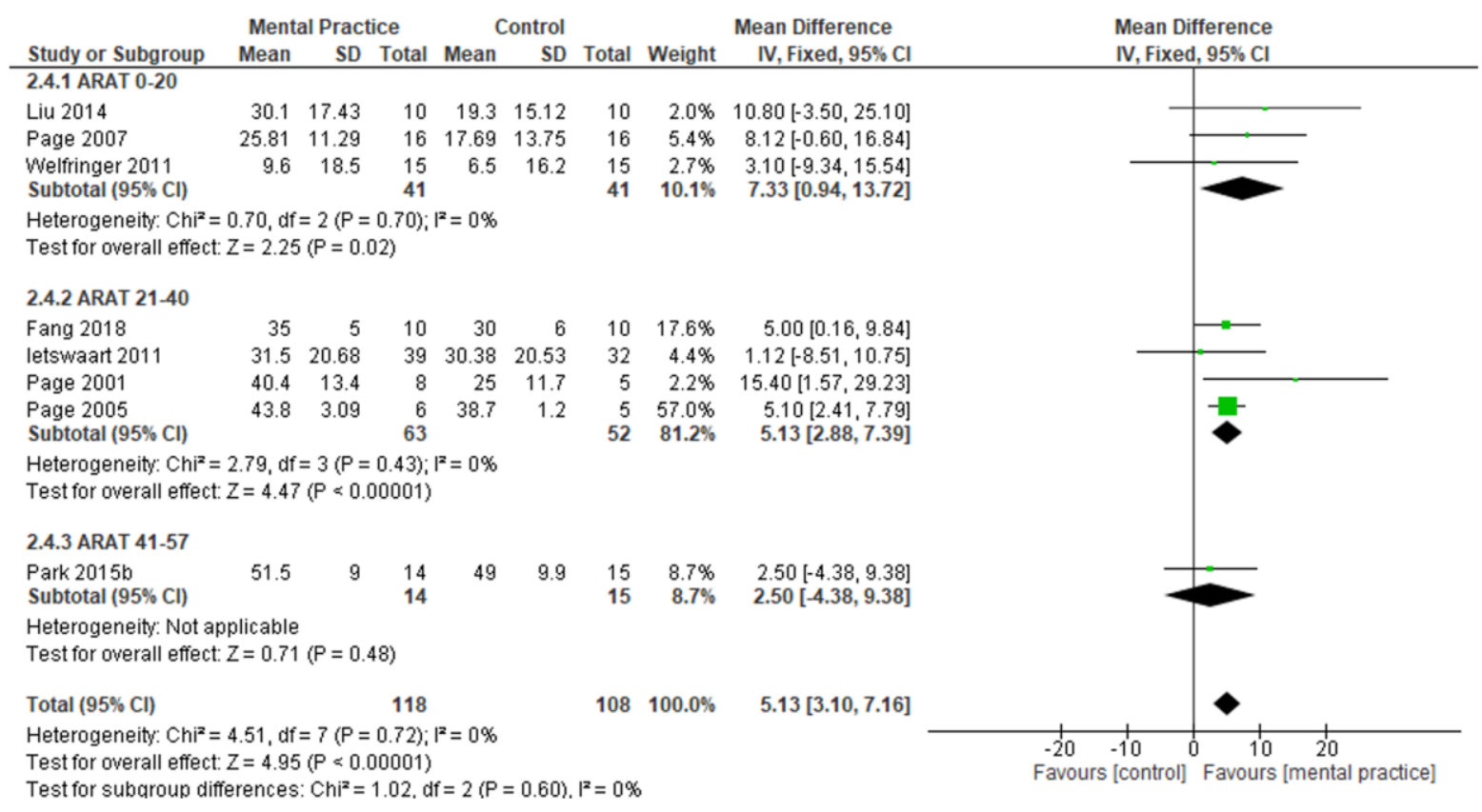

713 Figure 5

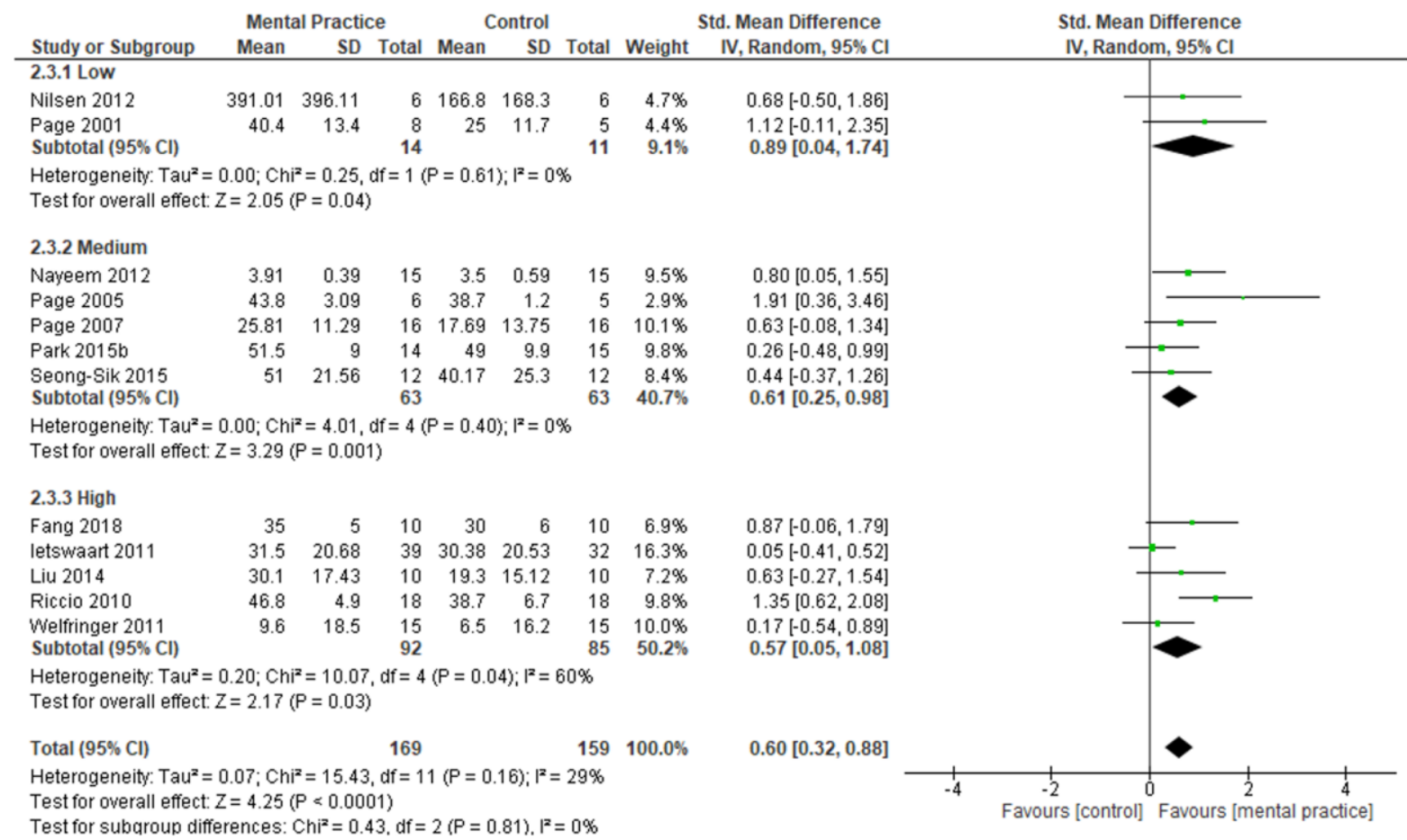

715 Legend: Data in Nilsen et al (2012) are reversed so that improvement is indicated by a 